\title{
Determination of Abundance of Different Groups of Mites in Relation to Abiotic Factors in Agro-Ecosystem
}

\author{
Muhammad Nabeel Saleem* \\ Department of Entomology, University of Agriculture Faisalabad, Pakistan
}

*Corresponding Author: Muhammad Nabeel Saleem, department of Entomology, University of Agriculture Faisalabad, Punjab, Pakistan.

Received: July 02, 2019; Published: August 06, 2019

DOI: 10.31080/ASAG.2019.03.0600

Soil mites community is generally well thought out as a useful biomarker of ecosystem disturbance, changes and other various types of conditions. Edaphic and environmental variables affect abundance and structure of soil mite community. The soil mite diversity, population dynamics are regulated by these factors and also play a significant role in decomposition and predation. This study was directed to figure out the fluctuations in seasonal abundance of soil mite community in an agro- ecosystem and to find out different abiotic factors in cotton, maize and wheat fields. Soil samples were taken on fortnightly basis during each crop season from Entomological Research Area, Square No. 3, University of Agriculture Faisalabad Pakistan. Soil mites were extracted using a locally made Macfayden-Tullgren Apparatus. Soil mite abundance was then correlated with different abiotic factor. No significant difference were found in soil mite abundance across all field sites. A total 751 soil mite individual were extracted out of which $51 \%$ belonged to Oribatida followed by $15 \%$ Prostigmata, 13\% ungrouped, $12 \%$ Astigmata, 6\% Mesostigmata and 5\% Gamasida. Average abundance per month was highest in summer month as compared to autumn, winter and spring. Lower rank abundance modeling revealed that the oribated mites contributes the most to soil mite fauna. Analysis of variance of abundance of sampling site showed that abundance was significantly different $(\mathrm{p}<0.05)$ except maize and wheat. Shannon diversities were not significantly different. All soil mites grouped occurred frequently in the samples. Cluster analysis grouped soil mites into three categories with reference to their abundance i.e. least, moderately and highly abundant. Abundance was positively co related with soil temperature, while it was negatively co related with soil moisture, soil pH and rainfall, while no co relation was observed with the soil organic matter. Principal component analysis that there was no specific co relation between all group composition and sampling period from April- Oct has entirely different species composition than sampling period from Nov-March. Correspondence analysis showed that distribution of relative abundance of all groups is quite dissimilar across the sampling period. Redundancy analysis showed that $\mathrm{pH}$, soil moisture and organic matter were highly co related with each other, the major group Oribatida showed strong negative co relation with relative humidity. to check significance of variables Monte-Carlo Permutation test was performed with 499 permutations. Step wise regression resulted in $\mathrm{R} 2$ value of $77 \%$ with three variables i.e. relative humidity, soil temperature ad soil organic matter. No serious auto correlation was found between residual after Durbin-Watson statistic was applied. Result revealed that abiotic factors especially soil temperature and relative humidity were major determinant for soil mite abundance.

Volume 3 Issue 9 September 2019 (C) All rights are reserved by Muhammad Nabeel Saleem. 